\title{
Contextual Emergence in the Description of Properties
}

\author{
Robert C. Bishop \\ Department of Philosophy, Rice University \\ Houston, Texas 77251, United States \\ Harald Atmanspacher \\ Institut für Grenzgebiete der Psychologie und Psychohygiene \\ Wilhelmstr. 3a, D-79098 Freiburg, Germany \\ Forthcoming in Foundations of Physics

\begin{abstract}
The role of contingent contexts in formulating relations between properties of systems at different descriptive levels is addressed. Based on the distinction between necessary and sufficient conditions for interlevel relations, a comprehensive classification of such relations is proposed, providing a transparent conceptual framework for discussing particular versions of reduction, emergence, and supervenience. One of these versions, contextual emergence, is demonstrated using two physical examples: molecular structure and chirality, and thermal equilibrium and temperature. The concept of stability is emphasized as a basic guiding principle of contextual property emergence.
\end{abstract}

Key Words: contextual emergence, contextual observables, molecular shape, temperature, topology, stability 


\section{Introduction}

A basic strategy for the scientific description of any system, physical or otherwise, is to specify its state and the properties associated with that state, and then introduce their evolution in terms of dynamical laws. This strategy presupposes that the boundary of a system can be defined with respect to its environment, although such a definition is often problematic. If it can be achieved, there is usually more than one possibility for specifying states and properties. The fact that states and properties can be formally and rigorously defined in fundamental physical theories distinguishes the structure of such theories as particularly transparent. A paradigmatic framework for a fundamental theory in present-day physics is quantum theory.

The situation is different in physical theories which are not regarded as fundamental (such as thermodynamics), or in descriptive approaches beyond physics (such as chemistry, biology or psychology). Though states and associated properties are often precisely defined in such theories, these descriptions are typically considered as less fundamental and as lacking the compact structure of fundamental theories. This circumstance provides one (though not the only) motivation for attempts to relate descriptions of systems, which are not fundamental in the sense mentioned above, to descriptions which are fundamental in this sense. The usual and (often too) simple framework in which corresponding relations are typically formulated is that of a hierarchy of descriptions. In a hierarchical picture (which can be refined in terms of more complicated networks of descriptions) there are higher-level and lower-level descriptions. More fundamental theories are taken to refer to lower levels in the hierarchy.

In such a simple framework, reduction and emergence are relations between different levels of descriptions of a system, its states and properties, or the (dynamical) laws characterizing their behavior. In the philosophical literature, the usual guiding idea behind reductionist approaches is to "reduce" higher-level features to lower-level features. In contrast, emergentist approaches emphasize the higher-level features by stressing the irreducibility of (some of) their aspects to lower levels. In this way, the emergence of features at higher levels is related to the emergence of novelty. Under the strong influence of positivist thinking in the first half of the 20th century, many philosophers enthusiastically welcomed the rapid and successful development of quantum theory as the ultimate justification for an entirely reductionist approach. For example, Reichenbach maintained that "today it is possible to say that chemistry is a part of physics, just as much as thermodynamics or the theory of electricity" (1978, p. 129), and Oppenheim and Putnam argued for "the possibility that science may one day be reduced to microphysics (in the sense in which chemistry seems today to be reduced to it...)" (1958, p. 35). Or consider Nagel's claim that "certain parts of nineteenth century chemistry (and perhaps the whole of this science) is reducible to post1925 physics" (1961, p. 362).

Such statements are not limited to philosophers. Dirac wrote that "... the underlying physical laws necessary for the mathematical theory of a large part of physics and the whole of chemistry are thus completely known, and the 
difficulty is only that the exact application of these laws leads to equations much too complicated to be soluble" (1929, p. 714). And Feynman et al. celebrated the Schrödinger equation as "... one of the great triumphs of physics. By providing the key to the underlying machinery of atomic structure it has given an explanation for atomic spectra, for chemistry, and for the nature of matter" (1965, p. 18f).

These and similar claims by eminent scientists have generated a lot of momentum for the belief that chemistry is reducible to quantum physics. However, there are serious problems with this belief. One example is that quantum mechanics does not allow the correct derivation of the periodic table (Löwdin 1969; Scerri 1998, 1999). Another example is the problem of deriving the nonlinear differential equations of chemical kinetics from the linear Schrödinger equation (Primas 1991, p. 163). Yet another example, which will be discussed in detail below, is the problem of how molecular structure is related to quantum mechanics, a problem first recognized by Heisenberg (1930).

Beyond the relationship of chemistry to quantum mechanics there are questions as to reductionism even among theories in physics. For instance, it is a fabled reductionist legend that thermodynamics can be reduced to lower-level physics descriptions. Thermodynamical phases such as liquidity (e.g., of water) cannot be strictly (i.e., without further assumptions) derived from the properties of individual (e.g., $\mathrm{H}_{2} \mathrm{O}$ ) molecules. Another example we discuss in more detail below concerns the alleged reduction (Kemeny and Oppenheim 1956; Nagel 1961) of temperature as a thermodynamical property to the kinetic energy of molecules.

The detailed discussion of these two examples will be used to exemplify the sophisticated way in which properties at one level of description are often related to properties at another level. While reductionists would argue that both necessary and sufficient conditions for higher-level properties are already present in the lower-level theory, it will be demonstrated that this is false in both of these examples, and presumably false in many others as well. In other words, there are properties of the higher-level theories (chemistry and thermodynamics) for which the full arsenal of the fundamental theories (quantum mechanics and statistical mechanics) provide no sufficient conditions for their derivation or definition.

Our examples illustrate the significance of a rarely discussed kind of interlevel relation we call contextual property emergence. We propose this category embedded in a framework classifying the relations between properties at different levels of description in section 2. It allows us to reconsider standard characterizations of property emergence in the light of modern developments in physics (sections 3 and 4). Guiding principles for contextual property emergence will be emphasized in section 5, which might be helpful for applications beyond physics. Specifically, one may think of relations between different levels of descriptions in brain physiology, e.g., relations between properties of neural ensembles or populations and properties of individual neurons and synapses. An even more ambitious application would be to the emergence of the mental from the physical. 


\section{Reduction and Emergence}

Reduction and emergence are used in a variety of senses in the literature. For the sake of simplicity, reduction and emergence schemes are typically organized in a hierarchical manner, such that levels of description or levels of reality are related to each other. As mentioned above, an analysis in terms of hierarchical levels often oversimplifies the picture. In general, non-hierarchical frameworks including other notions such as those of domains of description or domains of reality might be more appropriate.

As indicated by the distinction between levels of description and levels of reality, there is a difference between epistemological and ontological frameworks for reduction and emergence. Broadly speaking, descriptive terms are usually taken to be subjects of epistemological discourse while elements of reality are usually taken to be subjects of ontological discourse. Both types of discourse are used in reductionist and emergentist approaches. The concept of reference establishes a connection between descriptive terms and described elements of reality (leaving aside difficult questions about reference itself).

The distinction between epistemological and ontological discourse is not sufficient to exhaust the different ways in which the notions of reduction and emergence are used. In addition, it is also important to distinguish between different types of features which are to be related to others. There are three main categories of relations: theories/laws to other theories/laws, properties to other properties, and wholes to parts. Clearly, relations between theories/laws are predominantly epistemological. The relation between wholes and parts, on the other hand, is primarily conceived ontologically insofar as it refers to elements of reality rather than their description. In the literature on property relations, both epistemological and ontological frameworks can be found. Property relations are sometimes meant ontologically (i.e., regarding properties of elements of reality) and sometimes epistemologically (i.e., regarding descriptive terms referring to properties of elements of reality). A standard candidate for discussing epistemological theory relations is the relation between special relativity and classical mechanics.

A standard candidate for discussing ontological part/whole relations is the relation between molecules on the one hand and nuclei and electrons on the other. A standard candidate for discussing ontological property relations is the relationship between thermodynamic properties such as temperature and mechanical properties such as momenta; its epistemological variant would be the relationship between the descriptive terms referring to those properties. In this essay, we will mainly focus on epistemological property relations: reduction and emergence in the description of properties. ${ }^{1}$

In order to clearly distinguish between different concepts of reduction and emergence, it is desirable to have a transparent classification scheme so that their basic characteristics can be discussed coherently. A useful approach to-

\footnotetext{
${ }^{1}$ As the discussion in sections 3 and 4 will show, the relationships between properties at different levels lead to nontrivial ontological questions. We will not address these here, since their thorough discussion exceeds the scope of this essay.
} 
ward such a classification is based on the role which contingent contexts play in reduction and emergence. More precisely, the way in which necessary and sufficient conditions are assumed in the relation between different levels of description can be used to distinguish four classes of relations:

(1) The description of properties at a particular level of description (including its laws) offers both necessary and sufficient conditions to rigorously derive the description of properties at a higher level. This is the strictest possible form of reduction. As mentioned above, it was most popular under the influence of positivist thinking in the mid-20th century.

(2) The description of properties at a particular level of description (including its laws) offers necessary but not sufficient conditions to derive the description of properties at a higher level. This version, which we propose calling contextual emergence, indicates that contingent contextual conditions are required in addition to the lower-level description for the rigorous derivation of higher-level properties.

(3) The description of properties at a particular level of description (including its laws) offers sufficient but not necessary conditions to derive the description of properties at a higher level. This version includes the idea that a lower-level description offers multiple realizations of a particular property at a higher level-a feature characteristic of supervenience.

(4) The description of properties at a particular level of description (including its laws) offers neither necessary nor sufficient conditions to derive the description of properties at a higher level. This represents a form of radical emergence insofar as there are no relevant conditions connecting the two levels whatsoever.

Note that class (2) can complement class (3), so that it can be reasonable to say that higher-level features both supervene on and emerge from lower-level properties. This will be exemplified in section 4.2.

For obvious reasons, class (4) is unattractive if one is interested in explanatory relations between different levels of description. Non-reductive property dualism (e.g., Davidson 1980) would be an example of radical emergence. By contrast, class (1) is extremely appealing if one is interested in simple explanations. So-called "received views" of reduction-as Batterman (2002) refers to them-fall into this class. Unfortunately, many examples in the literature originally claimed to exemplify class (1) turn out to fail upon closer inspection (we discuss two such examples in section 4). Hence, there are fewer advocates of class (1) today, a notable exception being $\operatorname{Kim}(1998,1999)$.

As far as Nagel's (1961) influential work on reduction is concerned, two particular variants of his notion of reduction fit into class (1). One of them is sometimes called "homogeneous reduction" and reflects the (trivial) case in which the terms of the higher-level description are a subset of those of the lower-level description. In "heterogeneous reduction", there are connectability 
conditions ("bridge laws") connecting the two levels. If these bridge laws are contained in the lower-level description, the situation matches class (1). If this is not the case, terms of the lower-level description may be either necessary (2) or sufficient (3) for terms of the higher-level description.

In this sense, classes (2) and (3) are viable schemes for analyzing relationships between different levels of description. Supervenience relations, generally belonging to class (3), ${ }^{2}$ have been extensively discussed on the basis of Kim's proposals (Kim 1993). ${ }^{3}$ Interestingly, Kim himself has recently argued that supervenience may be inadequate for capturing relations in the sciences (Kim 1998, 1999). This development has led to an emphasis on realization relations (e.g., Kim 1998, 1999; Crook and Gillett 2000; Gillett 2002). In an epistemological interpretation, such relations fall into classes (1) - (3). Their ontological implications remain unaddressed in the present essay.

In the remainder of this essay, we will focus our discussion on class (2), contextual property emergence, which is less rigid than the philosophical notion of reduction on the one hand and provides more structure for interlevel relations than radical emergence on the other. In some respects, one might interpret our approach as representing a way of introducing effective techniques for generating bridge laws within the framework of class (2), but we will not pursue this viewpoint here. As will become clear below, our proposed scheme has much in common with a notion of reduction which is different from its standard philosophical meaning and has been distinguished as a physicist notion (Nickles 1973; Batterman 2002, pp. 17-19).

A "philosopher's notion" of property reduction, which attempts to specify both necessary and sufficient conditions for ensuring the derivation of higherlevel properties from the lower-level description alone, falls into our class (1). By contrast, the "physicist's notion" of property reduction intends to specify the necessary and sufficient conditions for the existence of some limit of an appropriate parameter at the lower level, which leads to the introduction of higher-level properties (e.g., the properties of a physical system in special relativity reduce to their Newtonian mechanics counterparts in the limit $v^{2} / c^{2} \rightarrow 0$, where $v$ is the system velocity and $c$ is the speed of light in vacuum). If necessary and sufficient conditions for the existence of the limit can be exhaustively specified within the lower-level description (e.g., special relativity) alone, then the physicist's notion of reduction is analogous to our class (1). As we discuss in detail below, however, the mentioned limit usually depends on conditions which can-

\footnotetext{
${ }^{2}$ Some versions of supervenience require that changes in lower-level descriptions of properties are both necessary and sufficient to bring about changes in a higher-level description of properties. Such versions are indistinguishable from reduction (Kim 1998) and fall into class (1).

${ }^{3}$ Recall that the British emergentists drew a distinction between resultant and emergent properties (see, e.g., McLaughlin 1982; Gillett 2003). The model for a resultant property was the process of vector addition of forces in classical particle mechanics, where the total length and direction of a force vector is the sum of its components. Properties that could be analyzed in such fashion were said to be resultant; they are reducible in the sense of class (1). Properties unanalyzable in such fashion were said to be emergent but can potentially refer to classes $(2)-(4)$.
} 
not be exhaustively specified at the lower level. In order to delineate contextual property emergence from other kinds of emergence, note that Kim (1999, pp. 19-22) characterizes the standard version of property emergence in terms of the following four conditions:

(A) Emergent properties at a higher level arise out of properties and relations characterizing the entities and properties at a lower level.

(B) Emergent properties are unpredictable, even given exhaustive information concerning the lower level.

(C) Emergent properties are inexplicable/irreducible by/to lower-level properties.

(D) Emergent properties have novel causal properties irreducible to the causal efficacy of lower-level properties.

In the next section, where we sketch a formal way to represent contextual property emergence, we will also analyze Kim's conditions (A) - (D) in more detail and demonstrate how they can be (re)formulated more precisely. As mentioned above, we will focus our attention on the epistemological interpretation of these conditions.

\section{Contextual Topologies and Asymptotic Expan- sions}

A first precondition for achieving a formal relation between descriptions at different levels is a well-defined concept of states and properties of the system considered at those levels. The algebraic approach in physics offers just such well-defined concepts. For example, in algebraic quantum theory, properties are introduced as so-called observables forming a $C^{*}$-algebra ${ }^{4} \mathcal{A}$ over the complex numbers which is not commutative in general. The associated concept of a state is introduced in terms of positive normalized linear functionals on $\mathcal{A}$. The state space of a fundamental theory in physics is chosen such that only the most basic assumptions are required for its definition. In other words, the state space is chosen as context-independent as possible. For instance, in quantum theory the state space used to represent states is standardly taken to be a Hilbert space endowed with the norm topology.

Contexts are contingent conditions referring to the degree of "abstraction" at which a theoretical framework is formulated. Each description requires "abstracting from," meaning disregarding those details of a given system (and its environment) which are to be considered irrelevant. Needless to say, declaring particular features as irrelevant is not a universally prescribed procedure,

\footnotetext{
${ }^{4} \mathrm{~A}{ }^{*}$-algebra is an algebra admitting an involution ${ }^{*}: \mathcal{A} \rightarrow \mathcal{A}$ with the usual properties. A *-algebra is normed, if there is a mapping $\|\|:. \mathcal{A} \rightarrow \mathbf{R}_{+}$with the usual properties. A complete normed *-algebra is a Banach *-algebra. A $C^{*}$-algebra is a Banach *-algebra $\mathcal{A}$ with the additional property $\left\|x^{*} x\right\|=\|x\|^{2}$ for all $x \in \mathcal{A}$ (see Takesaki 2002, chap. I.1).
} 
but must be tailored to particular purposes or interests. Features which are irrelevant in a particular context may be highly relevant in another.

For instance, temperature is an example of a feature that is relevant in thermodynamics but irrelevant in Newtonian or statistical mechanics. Light rays are relevant in geometric optics, but they are irrelevant in Maxwell's electrodynamics. The chirality of molecules is relevant in physical chemistry, but it is irrelevant in a Schr'odinger-type quantum mechanical description. Nevertheless, there are strategies for implementing the contexts due to which temperature is relevant in thermodynamics, due to which rays are relevant in geometric optics, and due to which chirality is relevant in physical chemistry, at the level of statistical mechanics, of electrodynamics, and of quantum mechanics, respectively.

A natural way to represent contexts of these kinds is the modification of the original topology of the lower-level state space into a contextual topology (Primas 1998). The finest topology corresponds to the most fundamental context, e.g. given by "first principles," and coarser topologies ${ }^{5}$ represent an increasing amount of contextual information not encoded in first principles. ${ }^{6}$ The key idea of relating properties at different levels of description to each other is to specify the difference between the descriptions in terms of the topologies of their corresponding state spaces.

Implementing a particular set of contexts as a contextual topology is usually nontrivial. A powerful tool often used for this purpose are asymptotic expansions (see Friedrichs 1955; Dingle 1973; Berry 1994; Batterman 2002). In order to formulate such an expansion, a reference state, which represents essential features of the context, has to be specified in the lower-level state space of the fundamental description. Examples for such reference states are Kubo-MartinSchwinger (KMS) states in statistical mechanics or electronic ground states of a molecule in quantum chemistry.

If the expansion is singular, it is not uniformly convergent in the intrinsic, fine topology of the fundamental description as an appropriate parameter tends to some limit. This discontinuous limiting behavior indicates the need for a change of topology. Examples for such parameters are the number of degrees of freedom for thermodynamics (thermodynamic limit), the wavelength for geometric optics (short-wavelength limit), or the electron mass divided by nuclear mass for physical chemistry (Born-Oppenheimer limit).

The crucial point then is to identify a new topology which regularizes the expansion such that it converges. Such a regularization is often possible by introducing a hierarchy of separable time scales, and then considering the motion of the system on "fast" time scales relative to almost fixed reference states according to "slow" time scales (for examples see Batterman 2002; Primas 1998). The separation of time scales leads to a coarser, contextual topology of the

\footnotetext{
${ }^{5}$ Consider the set of points $\Omega$ and a closure function which assigns to each set $\varphi \subset \Omega$ a set $\bar{\varphi} \subset \Omega$ (called the closure of $\varphi$ ) satisfying the properties $\varphi \subset \bar{\varphi}, \overline{\varphi \cup \psi}=\bar{\varphi} \cup \bar{\psi}, \bar{\oslash}=\oslash$, and $\overline{\bar{\varphi}}=\bar{\varphi}$. A topology with the closure function $\varphi \rightarrow \bar{\varphi}$ is finer than that given by the closure function $\varphi \rightarrow \overline{\varphi^{\prime}}$, if $\varphi \subset \overline{\varphi^{\prime}}$ for each $\varphi \subset \Omega$. The topology is coarser if $\varphi \supset \overline{\varphi^{\prime}}$ for each $\varphi \subset \Omega$.

${ }^{6}$ A powerful example of a first principle is symmetry. A detailed discussion of the relation between Primas' approach and symmetry principles has been given by Mainzer (1996).
} 
state space (see, e.g., Schulman and Gaveau 2001) providing a partition into equivalence classes of states. The new, coarser topology is compatible with the original, finer topology if they (in particular, their dynamics) are topologically equivalent with each other. However, the contextual topology is contingent in the sense that it is not given by the original finer topology or any other elements of the fundamental theory. The description in the coarser, contextual topology allows us to define new context-dependent features (novel properties) of a higherlevel description that are not defined in the original state space under the finer topology (cf. Primas 1998; Schulman and Gaveau 2001).

The procedure described so far represents a formal approach for a contextual emergence of higher-level properties on the basis of contexts in addition to the terms of the lower-level description. Qualitatively new, emergent properties, unavailable in the lower-level description, manifest themselves within the higher-level description. In somewhat less technical terms, the new state space is coarse-grained in a way allowing the definition of new (symbolic) states, together with associated observables, represented by the cells of its partition (cf. beim Graben 2004; Gaveau and Schulman 2005; Atmanspacher and beim Graben forthcoming). In this terminology, the choice of a proper partition is not prescribed at the lower-level description, but depends on the purpose of the partitioning and is usually based on properties that are foreign to the lower-level description. This is, for example, the core idea in the construction of a symbolic dynamics within the theory of dynamical systems (see Lind and Marcus 1995; beim Graben 2004).

Invoking a new contextual topology, then, accommodates novel properties as such within a higher-level description rather than approximating them as a limiting case in the topology of a lower-level description. It is important to realize that "the task of higher level descriptions is not to approximate the fundamental theory but to represent new patterns" (Primas 1998, p. 87). In general, these patterns are not reducible to a more fundamental level in the strict sense of class (1). Such reducibility would mean that only the first principles of the fundamental description are needed to describe new patterns exhaustively. If higher-level contexts in addition to first principles must be considered in order to rigorously derive descriptions of these new patterns so that reduction according to class (1) fails.

In such cases, contexts are at least as important as first principles. Given the fundamental theory and a suitable contextual topology, novel properties can be rigorously derived using asymptotic expansions that are regularized in the new contextual topology. Such higher-level descriptions in general cannot be considered as sub-descriptions of the fundamental theory because that theory alone does not imply the new contextual topology. In this sense we suggest considering emergent properties within class (2) of the proposed classification scheme. The contextual emergence of such properties can be physically well motivated and made mathematically rigorous via contextual topologies. Clearly, then, condition (A) above must be understood such that emergent properties arise from lower-level properties if a new contextual topology, not specified by the lower level theory, is additionally introduced. While necessary conditions for 
emergent properties exist at the lower level, the sufficient conditions represented by contexts do not exist at the lower level.

With respect to condition (B), emergent properties cannot be predicted from the lower level alone, even if exhaustive information concerning this level is assumed. Only the lower-level description plus the appropriate contextual topology renders emergent properties predictable as elements of a new algebra of observables. Similarly for condition (C): although even exhaustive information concerning the lower-level properties cannot yield an explanation of emergent properties, the emergence of new properties can be completely explained if the appropriate contextual topology is taken into account. This is at variance with the received view of explanation as exemplified by Hempel (1965).

Regarding condition (D), some further elaboration is necessary. Kim and others have argued that if new "causal powers" emerge at a higher level not reducible to or realized by lower-level properties, then we are left with at best a mystery as to where such "powers" come from or, at worst, the causal closure of the physical is violated (e.g. Kim 1998, 54-5; van Gulick 2001). As a first comment, the term "causal power" is somewhat vague, perhaps because the intuitive notion associated with this term is too imprecise or may be too broad. One may therefore prefer to speak about properties acting as constraints to bring about effects ("causal efficacy"). Second, since the new contextual topology allows for the prediction and explanation of emergent properties, based on a physically well-motivated new algebra of observables associated with the new contextual topology, the causal efficacy of these properties is considerably demystified and may even become intelligible (see below).

A reductionist might object that lower-level descriptions in fact provide derivable and predictable higher-level properties, albeit through very sophisticated, complex mathematical manipulations. For example Kim argues that the issue of whether a higher-level property is predictable from the lower level surely does not turn on when the mathematical relation between the two levels is simple rather than complex (Kim 1999, pp. 7-8). So one might worry that the level of mathematical sophistication involved in asymptotic analysis is somehow obscuring the reductive relations between the various levels, meaning that there is no need to relax the assumption of an "absolute" ontology.

However, although asymptotic analysis is indeed sophisticated and complex, this objection misses the crucial point of asymptotic reasoning. Sophisticated mathematics alone is insufficient to yield the asymptotic models and theories of one level from those of another for the examples we discuss. In addition, a suitably modified new contextual topology is required that is not given by the lower level, but must be implemented based on the context of the considered situation. As described above, there is a general systematic framework for defining a contingent contextual topology and then using that new topology along with the fundamental theory to rigorously derive a new, higher-level description. The crucial point is that the specific contextual topology needed to introduce emergent properties depends on contexts not given by the lower-level description. In contrast to radical emergence as in class (4), the contextual properties of higher-level descriptions emerge in a well-defined manner rather 
than "magically out of nowhere."

Our approach shares some similarities with Batterman's (2002) recent account of asymptotic emergence. He also indicates that conditions (A) - (C) must be suitably refined to understand how lower-level or "first principles" descriptions play their role in the prediction and explanation of emergent properties. While Batterman emphasizes the "no man's land" in the asymptotic region between theories (2002, pp. 115-120), we emphasize the role of contextual topologies in the prediction and explanation of emergent properties. As a more substantial point of difference, Batterman explicitly suggests restricting the discussion about emergent causal properties (condition (D)) to the role emergent properties play in asymptotic explanations (2002, pp. 126-29). In our judgment, this is a severe limitation that leaves important topics central to the debates unaddressed (e.g., how causal properties arise, why they are robust and efficacious, etc.). Our view allows for discussion of possible forms of downward causation and causal autonomy of emergent properties with respect to lower-level properties (see below).

\section{Examples}

This section discusses two examples of contextual emergence in a way which is detailed enough to see how contexts can be introduced leading to contextual topologies and emergent properties. Moreover, it is shown how necessary conditions for the emergence of novel properties are related to lower-level descriptions, whereas contingent contexts, not available within the lower-level description, represent sufficient conditions leading to well-defined properties at higher-order levels of description. ${ }^{7}$

\subsection{Molecular Structure and Chirality}

An essential ingredient of molecular physics and chemistry is the concept of molecular structure (shape, or "gestalt"), i.e. the configuration of the nuclei of the molecule. It is impossible to derive molecular structure from quantum mechanical first principles alone because in a complete quantum mechanical description electrons and nuclei are in entangled states. At the level of Schrödinger's equation, systems which differ by their molecular structure alone cannot be distinguished. A well-known example is different enantiomeric species of chiral molecules, whose chirality (handedness) is not a relevant observable at the level of quantum mechanics. Chirality is a classical observable, i.e., it is not an element of the algebra of observables of standard quantum mechanics (cf. Amann 1988).

As Woolley points out, the "systematic application of quantum mechanics to a molecule does not lead ... to the usual, and undoubtedly essentially correct, description of chemical phenomena that is obtained from orthodox quantum

\footnotetext{
${ }^{7}$ See Primas (1998) for more details and additional illustrative examples.
} 
chemistry" (1976, p. 31). The common reductionist argument for this failure is that the application of the fundamental laws of quantum mechanics to molecular systems leads to equations too complicated to solve; therefore, approximations are developed that supposedly "stand in" for the fundamental equations. ${ }^{8}$ However, this argument is inappropriate. The approaches used in quantum chemistry cannot be directly derived from fundamental equations of quantum physics, but, rather, appear to be strategies and solutions corresponding to different equations, where "new concepts which cannot be inferred from the underlying fundamental laws are required" (Woolley 1976, p. 32). Molecular structure is just such a concept and plays a crucial role in molecular physics and chemistry.

The concept of molecular structure has a long history (cf. Mainzer 1997). It was first precisely defined by Born and Oppenheimer (1927) through the famous so-called Born-Oppenheimer "approximation." Mathematically it represents an expansion in the parameter $\epsilon=\left(m_{e} / m_{n}\right)^{\frac{1}{4}} \rightarrow 0$, where $m_{e}$ is the electron mass and $m_{n}$ is the nuclear mass. In this limit the correlations among nuclei and electrons are suppressed and molecular structure can be addressed. Physically the limit $\epsilon \rightarrow 0$ represents a stability condition, where the electronic motion takes place against the background of a fixed nuclear frame. (Similarly, this can be achieved in the "adiabatic approximation" in which electrons are considered to move much faster than the nuclear frame of a molecule.) Nuclear permutation and rotational symmetries of the Hamiltonian at the fundamental level of Schrödinger's equation are also suppressed in the limit $\epsilon \rightarrow 0$ (Woolley 1976, p. 34). For a chemical level of discussion, such symmetries are inapplicable. Chemists typically measure systems in mixed states which lack these symmetries.

With respect to molecular structure, "no amount of computational investigation of the full molecular Schrödinger equation could lead to, for example, the familiar chemical idea of ammonia as a pyramidal molecule which is inverting, because the two modes of description have nothing in common" (Woolley 1976, p. 32). This is to say that the fundamental description does not capture crucial features of molecules. In the light of these facts, the Born-Oppenheimer "approximation" is more than a mere mathematical manipulation. It replaces the basic quantum description with a new description generated by the singular limit $\epsilon \rightarrow 0$. This replacement corresponds to a change in the algebra of observables needed for the description of molecular phenomena-a change which yields chirality as a classical observable related to molecular structure (see, e.g., Primas 1983, pp. 335-341; Amann 1993).

Using the terminology of Section 3, the asymptotic expansion $\epsilon \rightarrow 0$ defines a context required to change the norm topology of the basic quantum mechanical Hilbert space description into a new contextual topology. Molecular structure involves classical observables (i.e., commuting variables) expressible in the new contextual topology, but not expressible in the original norm topology. In the conceptual framework of Section 2, this contextual topology, together with the

${ }^{8}$ This line of argument has been called the "proxy defense" by Hendry (1998). 
lower-level quantum mechanical description, represents sufficient conditions for the description of molecular structure which are not available under the original topology. However, the new contextual topology is a contingent condition, being implied by neither standard quantum theory nor the original norm topology. In order to construct the appropriate contextual topology, one must first know what the appropriate observables are. In the case of molecular structure, the appropriate classical observables are not elements of the original algebra of observables given by the fundamental theory. There are no classical observables in standard formulations of quantum mechanics, so these observables must be specified in terms of an additional context beyond the basic Hilbert space description. Once the new contextual topology is in place, the asymptotic expansion can be regularized and molecular phenomena can be accurately described (cf. Primas 1998). The appropriate contextual topology together with standard quantum mechanics then allows the rigorous derivation of molecular properties like structure. Necessary conditions due to the original topology of the basic description are not violated as the new contextual topology is consistent with (though not implied by) the original topology.

To put things another way, as pointed out a complete Schrödinger-level description of molecules would involve correlations between nuclei and electrons, nuclear permutation and rotation symmetries, and an algebra of observables lacking the classical observables relevant to molecular structure. Given these features of the theory, standard quantum mechanics neither predicts nor explains structural properties of real molecules. The laws and properties of the standard theory are insufficient to yield molecular structure, minimize the correlations, break the symmetries and modify the algebra of observables. A rigorous description of molecular structure may emerge at the chemical level of description when a suitable contextual topology is provided. They key point is that such a contextual topology is not given or implied by fundamental quantum mechanics since such a new topology is absent. The theory makes no claims about molecular structure. At most it can supply necessary conditions for the description of these features of real molecules. This is precisely the conceptual scheme of contextual emergence, where the emergent property is the chiral (or other) structure of a molecule.

Such emergent properties can act in a way that is neither contained in, nor derivable from, nor predicted by the fundamental quantum description alone. Although one might think of them as merely "descriptive" terms, they have real consequences which suggest some ontological significance for them. For example, molecular structure and chirality are crucial for the explanation of optical activity where particular materials, optical isomers, rotate plane-polarized light passing through it (Woolley 1976, p. 32). They are invoked in typical descriptions and interpretations of single biomolecule spectroscopy (Weiss 1999) and in the nanomechanical properties of molecules (Gimzewski and Joachim 1999; Smith et al. 1999). And they play a crucial role in understanding DNA, various diseases and medications (e.g., DeCamp 1989; Avertisov et al. 1991). Furthermore, molecular structure is important for chemical and biological self-assembly (e.g. Lehn 2002; Whitesides and Grzybowski 2002). 
Additionally, the asymptotic expansion $\epsilon \rightarrow 0$ leads to a description of molecular dynamics with a hierarchy of time scales. While terms proportional to $\epsilon$ and $\epsilon^{3}$ vanish, terms proportional to $\epsilon^{2}$ characterize vibrations of the nuclear frame and terms proportional to $\epsilon^{4}$ characterize rotations of the nuclear frame. This hierarchy is related to time-scale separations providing the basis for molecular spectroscopy. Electronic spectra are characterized by the time scale $\tau_{0} \sim t$, while vibrational spectra are characterized by the slower time scale $\tau_{2} \sim \epsilon^{2} t$ and rotational spectra are characterized by the even slower time scale $\tau_{4} \sim \epsilon^{4} t$. An interesting feature of this hierarchy is that adjacent levels are connected by feedback loops such that each lower level is subordinated to the next higher level. In this way, higher-level behavior constrains lower-level behavior. This concept is at variance with traditional reductive hierarchies but can be discussed in the context of downward causation.

A recent example of such downward causation in the formation of chiral molecules has recently been reported by Ribó et al. (2001). Typically, in the absence of external polarization or other influences, the orientation of chiral molecules is governed by random fluctuations with both orientations arising equally likely. However, using particular aqueous solutions far from thermodynamic equilibrium, Ribó et al. showed that stirring-a macroscopic processchanges the random distribution of orientations of chiral molecules. This can be described as a special type of symmetry breaking. In this sense, higher-level constraints influence the dynamics of molecular formation at a lower level. In other words, molecular structure can be influenced by operations at higher-order levels. In this context, one might have the general worry that there is a potential problem for causal exclusion or paradox. However, this problem arises only if one assumes that the causal efficacy of the lower-level description is exhaustive of all lower-level properties. This assumption is too strong, however. The dynamical equations of lower-level theories like quantum mechanics always require the addition of particular global constraints (often in the form of boundary conditions) in order either to form a well-posed problem or to remove degeneracies (i.e., the multiplicity of possible solutions). These constraints are usually not given at the lower level. In the experiments of Ribó et al. (2001), macroscopic constraints have downward causal effects on the formation of chiral molecules. In this example, higher-level constraints provided by the stability condition arising from the Born-Oppenheimer procedure remove degrees of freedom existing in the dynamical equations at the lower level, addressing the combinations of electrons and nuclei (such combinations are under determined by Schrödinger's equation).

\subsection{Thermal Equilibrium and Temperature}

A second, much discussed example is the reduction or emergence, respectively, of thermodynamic properties such as temperature to or from properties at lowerlevel descriptions. Although there are various approaches to discussing this issue (e.g. Haken 1983; Glansdorff and Prigogine 1971), we will focus on an algebraic reconstruction of the emergence of thermodynamic properties. The 
reason is that this point of view allows us to pose the problems in a way which is conceptually transparent (though mathematically involved; see section 3). The usual candidates of lower-level descriptions in this context are statistical mechanics and point mechanics. How are these levels of description related to thermodynamics?

To start with the less controversial issue, the step from point mechanics to statistical mechanics is essentially based on the formation of an ensemble distribution. This is to say that particular properties of a system are defined in terms of a statistical ensemble description (e.g., as moments of a many-particle distribution function) which refers to the state of an ensemble rather than to states of single particles in an individual description.

An example is the mean kinetic energy of a system of $N$ particles, which can be calculated from the distribution of the momenta of all particles. The expectation value of kinetic energy is defined in the limit of infinitely many particles, assuming the applicability of the usual limit theorems such as the law of large numbers. Any expectation value of a thermodynamic property (including temperature) whose definition is based on a statistical ensemble description presupposes (infinitely) many degrees of freedom, i.e., the thermodynamic limit $N \rightarrow \infty$.

The more controversial issue in discussing the reduction or emergence of temperature refers to the step from statistical mechanics to thermodynamics (cf. the discussion by Compagner 1989). It amounts to relating the expectation value of a momentum distribution of a many-particle ensemble to the temperature of the system as a whole. In many philosophical discussions it is argued that the thermodynamic temperature "of a gas is the mean kinetic energy of the molecules which by hypothesis constitute the gas" (Nagel 1961, p. 341). This statement suggests a fairly straightforward reduction of thermodynamic temperature to statistical mechanics.

Such a rough picture, however, would be a gross mischaracterization, based on a too generous treatment of some important details. In addition to the thermodynamic limit, thermodynamic descriptions presume thermodynamic, or briefly thermal, equilibrium as a crucial assumption which is neither formally nor conceptually available at the level of statistical mechanics. Moreover, the very concept of temperature is fundamentally foreign to statistical mechanics and has to be introduced, e.g., on the basis of phenomenological arguments. ${ }^{9}$

Thermal equilibrium is formulated by the zeroth law of thermodynamics: if two systems are both in thermal equilibrium with a third system, then they are said to be in thermal equilibrium with each other. (In this sense, the definition of temperature is relational.) Based on this equivalence relation, the phenomenological concept of temperature can be introduced in the usual textbook way. Since thermal equilibrium is not defined at the level of statistical mechanics, temperature is not a mechanical property but, rather, emerges as a novel property at the level of thermodynamics. In this sense, the concept of

\footnotetext{
${ }^{9}$ We do not discuss the phenomenological significance of the concept of temperature in terms of phenomenally experienced qualities of "warm" or "cold," which may also be considered in this context.
} 
thermal equilibrium serves as a context providing conditions for a proper discussion of temperature. This context is available at the higher-level description of thermodynamics. It can be recast in terms of a class of distinguished statistical states, the so-called Kubo-Martin-Schwinger (KMS) states, at the lower-level statistical description. These states are defined by the KMS condition, which characterizes the stability of a KMS state against local perturbations. The KMS condition essentially implements a higher-level context, the zeroth law of thermodynamics, in terms of a stability condition at the lower level of statistical mechanics. The second law of thermodynamics expresses this stability condition in terms of a maximization of entropy. (Equivalently, the free energy of the system is minimal in thermal equilibrium.) If a system is in a KMS state, then this state is the canonical Gibbs state, uniquely defining a parameter interpreted as a (inverse) temperature. ${ }^{10}$

In the framework of an algebraic statistical mechanics description, KMS states serve as reference states for a Gel'fand-Naimark-Segal (GNS) construction. Such reference states are functionals on a fundamental, lower-level, algebra of observables. They induce a new, contextual topology in the state space of statistical mechanics, which is coarser than the original topology and gives rise to another, higher-level algebra of observables including thermodynamic temperature as a property of the system. It has been shown in detail by Takesaki (1970) how temperature emerges as a classical observable from an underlying quantum statistical description. ${ }^{11}$ Temperature is then an element of an algebra $\mathcal{M}$ of contextual observables, where the context is introduced by the KMS state as a reference state plus the contextual topology induced by this reference state. Since mechanical descriptions are given by type I $W^{*}$-algebras and the contextual $W^{*}$-algebra $\mathcal{M}$ is of type III, ${ }^{12}$ temperature cannot be an element of a mechanical description (Primas 1998). Hence, temperature is not reducible to statistical mechanics in any straightforward sense.

Thermodynamic temperature is an example of a contextually emergent property, which is neither contained in nor predicted by the exhaustive lower-level mechanical description alone. However, given the lower-level mechanical description and an appropriate contextual topology (based on the KMS state), thermodynamic quantities can be rigorously derived. Again, the contextual topology is a contingent condition not implied by the lower-level theory with the original topology as neither the concepts of thermal equilibrium nor of KMS

\footnotetext{
${ }^{10}$ For more details concerning the conceptual significance of the KMS condition see Sewell (2002, chap. 5). The stability requirement imposed by the KMS condition is discussed in detail in Atmanspacher and beim Graben (2006).

${ }^{11}$ Another thermodynamical concept, which is based on the KMS condition in a similar way, is the chemical potential. It has been shown that chemical potential emerges as a classical observable from an underlying quantum statistical description as well (Müller-Herold 1980).

${ }^{12} \mathrm{~A} W^{*}$-algebra is a *-algebra which is isomorphic to a closed algebra of observables on a Hilbert space. A $C^{*}$-algebra $\mathcal{M}$ is a $W^{*}$-algebra if and only if it is the dual of a Banach space $\mathcal{M}_{*}$, where $\mathcal{M}_{*}$ is the predual of $\mathcal{M}$ (see Takesaki 2002 , Chap. III.3). $W^{*}$-algebrs can be classified by their central decompositions, i.e. by factors. A factor is of type I if it contains an atom. It is of type III if it does not contain any nonzero finite projection. It is of type II if it is atom-free and contains some nonzero finite projection. For more details see Takesaki 2002, p. 296).
} 
states are applicable at the lower level. This is precisely the conceptual scheme of contextual emergence, where the emergent property is the temperature (or other thermal features) of thermodynamics.

At this point let us emphasize that the contextual emergence of temperature does not exclude or contradict the possibility of conceiving temperature as supervenient. As supervenience is based on sufficient conditions at the lower-level description, it takes into account that different statistical mixtures (distributions) of particles can be considered as multiple realizations of a thermal state with the same temperature. The notion of contextual emergence addresses the question of how statistical properties are related to thermal properties for each individual statistical realization. Contextual emergence does not address issues of multiple realization but tries to elucidate principles which allow us to understand interlevel relations in each individual instantiation in a conceptually clear manner.

If crucial contextual details are not disregarded, one may even conceive of properties that can act as constraints for lower-level properties, emerging at higher levels of physical descriptions. For example, the behavior of individual particles may be constrained by the collective behavior of a many-particle system as a whole, e.g., described at the level of thermodynamics (see Bishop 2004, section 6).

\section{Guiding Principles for Contextual Emergence}

After the detailed discussion of the emergent properties of chirality and temperature as examples for contextual emergence, it is worthwhile addressing its key features. Repeating the general characterization given in section 2: the description of properties (and laws) at a particular level of description offers necessary but not sufficient conditions to derive properties at a higher level of description. In logical terms, the necessity of conditions at the lower level of description means that components of the higher level of description imply components of the lower level of description. The converse does not hold in contextual emergence, as the the lower-level description does not offer sufficient conditions for the derivation of higher-level components Additional, contingent conditions specifying the context for the transition from the lower to the higher level of description are required in order to provide such sufficient conditions.

In the example of chirality, the notion of molecular structure is the key context. Molecular structure is not available at a fundamental quantum mechanical level of description. Using the Born-Oppenheimer expansion as a context, i.e., considering the limit $\left(m_{e} / m_{n}\right)^{\frac{1}{4}} \rightarrow 0$, chirality can be obtained as an emergent property at the level of molecular descriptions. Mathematically speaking, this limit converges in the framework of a molecular description and leads to new, contextual properties, including chirality. Physically speaking, the convergence of the limit corresponds to a stability criterion establishing a nuclear frame with respect to which the motion of the electrons can be considered as separated. Since the quantum mechanical level of description is necessary to 
derive the higher-level property of chirality, quantum mechanical principles or laws cannot be violated by any higher-level description incorporating chirality. That the quantum mechanical level of description alone is not sufficient is recognized by the fact that it does not give rise to an algebra of observables including chirality unless additional contingent conditions are given.

In the example of temperature, the notion of thermal equilibrium is the key context. Thermal equilibrium is not available at the level of description of Newtonian or statistical mechanics. Implementing thermal equilibrium in terms of the KMS condition and considering the limit $\rightarrow \infty$ at the level of statistical mechanics, temperature can be obtained as an emergent property at the level of a thermodynamical description. Mathematically speaking, this limit converges in the framework of a thermodynamical description and leads to new, contextual properties, including temperature. Physically speaking, the convergence of the limit corresponds to a stability criterion for KMS states which is induced by the contextual condition of thermal equilibrium at the level of thermodynamics and implemented at the level of statistical mechanics.

Since the Newtonian and statistical mechanical levels of description are necessary to derive the high-level property of temperature, principles or laws of these levels of description cannot be violated by any higher-level description incorporating temperature. That the Newtonian and statistical mechanical levels of description alone are not sufficient is recognized by the fact that they do not give rise to an algebra of observables including temperature unless additional contingent contexts are specified and properly implemented.

The significance of contextual emergence as opposed to reduction in the discussed examples is clear. Of course, it would be interesting to extend the general construction scheme for emergent properties to other cases. More physical examples are indicated and discussed, for example, in Primas (1998) and Batterman (2002). We propose the concept of stability as a key principle for the construction of a contextual topology and an associated algebra of contextual observables in examples beyond physics.

One possible, and ambitious, case refers to emergent properties in the framework of cognitive neuroscience. A particularly active field of research here is concerned with the emergence of new properties at the level of neural ensembles from lower-level properties of individual neurons. Particular interest in this issue derives from the fact that cognitive capabilities are usually correlated with the activity of neural ensembles, but detailed neurobiological knowledge refers mainly to the properties of individual neurons. Closing the gap in our understanding of the relation between properties of neural ensembles and individual neurons could contribute significantly to understanding neurobiological correlates of consciousness.

As a possible framework for research in this area, the scheme of contextual emergence, as exemplified in the previous section, might be fruitfully applied as follows. Novel properties at the (higher) level of neural ensembles would have necessary but not sufficient conditions at the (lower) level of neurons. In order to identify contexts providing such sufficient conditions, those among the many possible ensemble properties which are relevant or interesting as emergent prop- 
erties must first be clarified. Assuming that stability criteria and associated time scales play a role analogous to physical examples, techniques of nonlinear dynamics for modeling ensembles in terms of attractors with particular stability properties and relaxation times or escape times suggest themselves. This can be implemented easily for powerful modeling tools such as neural networks (Anderson and Rosenfeld 1989) or coupled map lattices (Kaneko and Tsuda 2000).

Contextual emergence might even be a viable scheme for addressing relations between the neurobiology of the brain at various levels, on the one hand, and cognitive or psychological features, on the other-in other words, addressing the relation between material (brain) and mental (consciousness) features. In another paper (Atmanspacher and beim Graben forthcoming) a number of corresponding aspects of cognitive neuroscience have been worked out in detail recently.

In that approach, mental states are considered to emerge from neural states by partitioning the neural state space. Well-defined mental states provide contexts inducing a criterion of structural stability for the neurodynamics which can be implemented by particular partitions and analyzed in terms of symbolic dynamics. These stability criteria are applied to the discussion of neural correlates of consiousness, to the definition of macroscopic neural states, and to aspects of the symbol grounding problem. In particular, it is shown that mental descriptions that are topologically equivalent to the neurodynamical description emerge if the partition of the neural phase space is generating.

\section{Summary and Concluding Remarks}

The goal of epistemological property reduction is to derive the description of higher-level properties exhaustively in terms of the description of properties at the most fundamental level of physical theory, no matter how remote these higher-level properties are from that most fundamental level. The implicit assumption in this program is that the description of all properties which are not included at the fundamental level can be constructed or derived from this level. The concept and the examples discussed in this essay pose serious difficulties for this program. Molecular structure is a novel property emerging from a more fundamental quantum mechanical description, but it is not derivable from the lower-level description alone.

Similarly, temperature is a novel property emerging from a more fundamental statistical mechanical description, but it is likewise not derivable from the lower-level description alone. The concept of contextual emergence addresses such situations properly. Contextual emergence is characterized by the fact that the description of lower-level properties provides necessary, but not suffi-

cient conditions for the description of higher-level properties. The presence of necessary conditions indicates that the lower-level description provides a basis for higher-level descriptions, while the absence of sufficient conditions means that higher-level properties are neither logical consequences of the lower-level 
description nor can such properties be rigorously derived from the lower-level description alone.

Hence, the notion of strong reduction is inapplicable in these cases. Sufficient conditions for a rigorous derivation of higher-level properties can be introduced through specifying contexts reflecting the particular kinds of contingency in a given situation. These contexts can be implemented as stability criteria in the lower-level description and induce a change in the topology of the corresponding state space (e.g., due to coarse-graining). There is, then, a mathematically well-defined procedure for deriving higher-level properties given the lower level description plus the contingent contextual conditions.

A key ingredient of this procedure is the definition of some type of stability condition (e.g., the KMS condition) based on considerations required to establish the framework of a higher-level description (e.g., thermal equilibrium). This condition is typically implemented as a reference state with respect to which an asymptotic expansion is singular in the lower-level state space. Its regularization defines a novel, contextual topology in which novel, emergent properties can be rigorously introduced. In the thermodynamic example, this procedure is represented by the GNS-construction.

Contextual property emergence and the associated identification of appropriate stability conditions may have applications in other domains such as biology and psychology, and, ultimately, in the relationship between the mental and the physical. For example, debates in the philosophy of mind discuss the physical either as being both necessary and sufficient (reduction) or as being only sufficient (supervenience) for the mental. Contextual emergence is located between those two positions. As worked out in detail elsewhere (Atmanspacher and beim Graben 2006), it attenuates reductive claims and complements (rather than contradicts) particular ideas about the supervenience of the mental on the physical.

Examples of contextual property emergence such as those discussed here can serve as models for generalizations concerning ideas related to ontological relativity and downward causation in broader contexts. Both notions have been addressed in recent work (Atmanspacher and Kronz 1999; Ribó et al. 2001; Bishop 2004), and they have been illustrated for examples from physics and chemistry. Eventually such a line of investigation might yield steps toward a better understanding of how mind can emerge in and have an influence on a physical world.

\section{Acknowledgments}

We are grateful to Carl Gillett, Peter beim Graben, Fred Kronz and Michael Silberstein for numerous discussions and useful comments. In addition, we appreciate the constructive criticism by anonymous referees which was instrumental for improving earlier versions of this paper. 


\section{References}

A. Amann, "Chirality as a classical observable in algebraic quantum mechanics," in Fractals, Quasicrystals, Chaos, Knots, and Algebraic Quantum Mechanics, A. Amann et al., eds. (Kluwer Academic Publishers, Dordrecht, 1988), pp. 305-325.

A. Amann, "The Gestalt problem in quantum theory: Generation of molecular shape by the environment," Synthese 97, 125-156 (1993).

J. A. Anderson and E. Rosenfeld, Neurocomputing: Foundations of Research (MIT Press, Cambridge, MA, 1989).

H. Atmanspacher and P. beim Graben, "Contextual emergence of mental states from neurodynamics," Chaos and Complexity Lett., forthcoming.

H. Atmanspacher and F. Kronz, "Relative onticity," in On Quanta, Mind and Matter: Hans Primas in Context, H. Atmanspacher, A. Amann, and U. Müller-Herold, eds. (Kluwer, Dordrecht, 1999), pp. 273-294.

V. Avertisov, V. Goldanskii and V. Kuz'min, "Handedness, origin of life and evolution," Physics Today 44(2), 33-41 (1991).

R. Batterman, The Devil in the Details. (Oxford University Press, Oxford, 2002).

M. Berry, "Asymptotics, singularities and the reduction of theories," Logic, Methodology and Philosophy of Science IX: Proceedings of the Ninth International Congress of Logic, Methodology and Philosophy of Science, Uppsala 1991, in D. Prawitz, B. Skyrms and D. Westerstahl, eds. (Elsevier, North-Holland, Amsterdam, 1994), pp. 597-607.

R. C. Bishop, "Nonequilibrium statistical mechanics Brussels-Austin style," Studies in History and Philosophy of Modern Physics 35, 1-30 (2004).

M. Born and R. Oppenheimer, "Zur Quantentheorie der Molekeln," Annalen der Physik 84, 457-484 (1927).

A. Compagner, A., "Thermodynamics as the continuum limit of statistical mechanics," Am. J. of Phys. 57(2), 106-117 (1989).

S. Crook and C. Gillett, "Why physics alone cannot define the 'physical'," Can. J. of Phil. 31, 333-360 (2001).

D. Davidson, Essays on Actions and Events. (Oxford University Press, Oxford, 1980).

W. H. DeCamp, "The FDA perspective on the development of stereoisomers," Chirality 1, 2-6 (1989). 
R. Dingle, Asymptotic Expansions: Their Derivation and Interpretation. (Academic Press, New York, 1973).

P. A. M. Dirac, "Quantum mechanics of many-electron systems," Proceedings of the Royal Society of London A123, 714-733 (1929).

R. Feynman, R. Leighton and M. Sands, The Feynman Lectures on Physics: Quantum Mechanics. (Addison-Wesley, Reading, MA, 1965).

K. Friedrichs, "Asymptotic phenomena in mathematical physics," Bulletin of the American Mathematical Society 61, 485-504 (1955).

B. Gaveau and L. S. Schulman, "Dynamical distance: Coarse grains, pattern recognition, and network analysis," Bulletin des Sciences Mathematiques 129, 631-642 (2005).

C. Gillett, "The varieties of emergence: Their purposes, obligations and importance," Grazer Philosophische Studien 65, 95-121 (2002).

C. Gillett, "Non-Reductive realization and non-reductive identity: what physicalism does not entail," in Physicalism and Mental Causation, S. Walter and H.-D. Heckmann eds. (Imprint Academic, Thorverton, 2003), pp. $31-57$.

J. Gimzewski and C. Joachim, "Nanoscale science of single molecules using local probes," Science 283, 1683-8 (1999).

P. Glansdorff and I. Prigogine, Thermodynamic Theory of Structure, Stability, and Fluctuations. (Wiley, New York, 1971).

P. beim Graben, "Incompatible implementations of physical symbol systems," Mind and Matter 2(2), 29-52 (2004).

H. Haken (1983), Synergetics. (Springer, Berlin, 1983).

W. Heisenberg, The Physical Principles of the Quantum Theory. (Dover, New York, 1930).

C. Hempel, Aspects of Scientific Explanation and Other Essays in the Philosophy of Science. (The Free Press, New York, 1965).

R. Hendry, "Models and Approximations in Quantum Chemistry," in Idealization IX: Idealization in Contemporary Physics, Poznan Studies in the Philosophy of the Sciences and the Humanities 63, N. Shanks, ed. (Rodopi, Amsterdam, 1998), pp. 123-142.

K. Kaneko and I. Tsuda, Complex Systems: Chaos and Beyond. (Springer, Berlin, 2000).

J. G. Kemeny and P. Oppenheim (1956), "On Reduction," Philosophical Studies 7, 6-19 (1956). 
J. Kim (1993), Supervenience and Mind. (Cambridge University Press, Cambridge, 1993).

J. Kim, Mind in a Physical World: An Essay on the Mind-Body Problem and Mental Causation. (MIT Press, Cambridge, MA, 1998).

J. Kim, "Making sense of emergence," Philosophical Studies 95, 3-36 (1999).

J.-M. Lehn, "Toward self-organization and complex matter," Science 295, 2400-2403 (2002).

D. Lind and B. Marcus, Symbolic Dynamics and Coding, (Cambridge University Press, Cambridge, 1995).

P.-O. Löwdin, "Some comments on the periodic system of elements," Int. J. of Quant. Chem. 3: 331-334(1969).

K. Mainzer, Symmetries of Nature. (DeGruyter, Berlin, 1996).

K. Mainzer, "Symmetry and complexity: fundamental concepts of research in chemistry," Hyle 3, 29-49 (1997).

B. McLaughlin, "British Emergentism," in Emergence or Reduction? Essays on the Prospects of Nonreductive Physicalism, A. Beckermann et al., eds. (Walter de Gruyter, Berlin, 1982), pp. 49-93.

U. Müller-Herold, "Disjointness of $\beta$-kms states with different chemical potential," Lett. in Math. Phys. 4, 45-48 (1980).

E. Nagel, The Structure of Science. (Harcourt, Brace \& World, New York, 1961).

T. Nickles, "Two concepts of intertheoretic reduction," J. of Phil. 70, 181-201 (1973).

P. Oppenheim and H. Putnam, "The unity of science as a working hypothesis," in Minnesota Studies in the Philosophy of Science vol. 2, H. Feigl, G. Maxwell and M. Scriven, eds, (University of Minnesota Press, Minneapolis, 1958), pp. 3-35.

H. Primas, Chemistry, Quantum Mechanics and Reductionism. (Springer, Berlin, 1983).

H. Primas, "Reductionism: palaver without Precedent," in The Problems of Reductionism in Science, E. Agazzi, ed., (Kluwer, Dordrecht, 1991), pp. 161-172.

H. Primas, "Emergence in exact natural sciences," Acta Polytechnica Scandinavica 91, 83-98 (1998). 
H. Reichenbach, "The aims and methods of physical knowledge," in Hans Reichenbach: Selected Writings 1909-1953, M. Reichenbach and R. Cohen, eds., (Reidel, Dordrecht, 1978), pp. 81-225.

J. Ribó, J. Crusats, F. Sagués, J. Claret and R. Rubires, "Chiral sign induction by vortices during the formation of mesophases in stirred solutions," Science 292, 2063-2066 (2001).

E. Scerri, "How good is the quantum mechanical explanation of the periodic system?" J. of Chem. Ed. 75, 1284-1285 (1998).

E. Scerri, "A critique of Atkins' periodic kingdom and some writings on electronic structure," Found. of Chem. 1, 297-305 (1999).

L. S. Schulman and B. Gaveau, "Coarse grains: the emergence of space and order," Found. of Phys. 31, 713-731 (2001).

G. Sewell, Quantum Mechanics and Its Emergent Macrophysics. (Princeton University Press, Princeton, 2002).

D. Smith, H. Babcock and S. Chu, "Single-polymer dynamics in steady shear flow," Science 283, 1724-1727 (1999).

M. Takesaki, "Disjointness of the kms states of different temperatures," Commun. in Math. Phys. 17: 33-41 (1970).

M. Takesaki, Theory of Operator Algebras I. (Springer, Berlin, 2002).

R. Van Gulick, "Reduction, emergence and other recent options on the mind/body problem," J. of Consc. Studies 8, 1-34 (2001).

G. Whitesides and B. Grzybowski, "Self-assembly at all scales," Science 295, 2418-2421 (2002).

S. Weiss, "Fluorescence spectroscopy of single biomolecules," Science $\mathbf{2 8 3}$, 1676-1683 (1999).

R. Woolley, "Quantum theory and molecular structure," Advances in Phys. 25: 27-52 (1976). 